\title{
Some Properties of Random Apollonian Networks
}

\author{
Alan Frieze and Charalampos E. Tsourakakis
}

Abstract. In this work, we analyze fundamental properties of random Apollonian networks [Zhang et al. 06, Zhou et al. 05], a popular random graph model that generates planar graphs with power-law properties. Specifically, we analyze the degree distribution, the $k$ largest degrees, the $k$ largest eigenvalues, and the diameter, where $k$ is a constant.

\section{Introduction}

Due to the surge of interest in social networks, the Web graph, the Internet, biological networks, and many other types of networks, a large amount of research has focused in recent years on modeling real-world networks. Existing well-known models include the preferential attachment model [Barabási and Albert 99], Kronecker graphs [Leskovec and Faloutsos 07], the Cooper-Frieze model [Cooper and Frieze 03], the Aiello-Chung-Lu model [Aiello et al. 01], protean graphs [Pralat and Wormald 07], and the Fabrikant-Koutsoupias-Papadimitriou model [Fabrikant et al. 02].

Color versions of one or more of the figures in the article can be found online at www.tandfonline.com/uinm. 


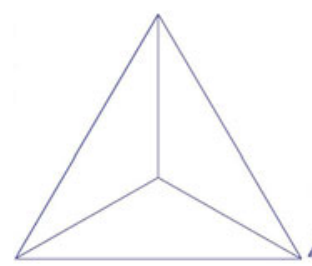

(a) $t=1$

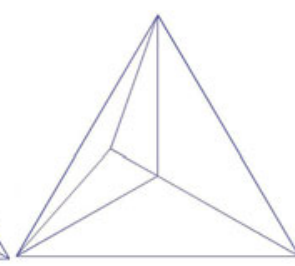

(b) $t=2$

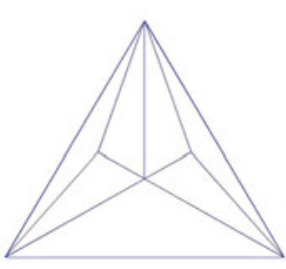

(c) $t=3$

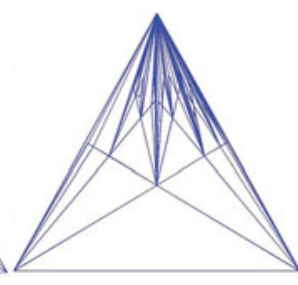

(d) $t=100$

Figure I. Snapshots of a random Apollonian network (RAN) at (a) $t=1$, (b) $t=$ 2 , (c) $t=3$, (d) $t=100$.

Despite the fact that the family of planar graphs is a particularly important family [Klein 14] and that it models important real-world graphs such as road networks, there exist few generators of real-world planar graphs.

In this work, we focus on random Apollonian networks (RANs), a popular random graph model for generating planar graphs with power-law properties [Zhou et al. 05]. Before we state our main results, we briefly describe the model.

An example of a RAN is shown in Figure 1. At time $t=1$, the RAN is shown in Figure 1(a). At each step $t \geq 2$, a face $F$ is chosen uniformly at random among the faces of $G_{t}$. Let $i, j, k$ be the vertices of $F$. We add a new vertex inside $F$ and connect it to $i, j, k$. Higher-dimensional RANs also exist that instead of triangles, have $k$-simplexes $k \geq 3$; see [Zhang et al. 06]. It is easy to see that the number of vertices $n_{t}$, edges $m_{t}$, and faces $F_{t}$ at time $t \geq 1$ in a RAN $G_{t}$ satisfy

$$
n_{t}=t+3, \quad m_{t}=3 t+3, \quad F_{t}=2 t+1 .
$$

Note that a RAN is a maximal planar graph, since for every planar graph, one has $m_{t} \leq 3 n_{t}-6 \leq 3 t+3$.

Surprisingly, despite the popularity of the model, various important properties have been analyzed experimentally and heuristically with insufficient rigor. In this work, we prove the following theorems using existing techniques [Alon and Spencer 08, Flaxman et al. 05, Mihail and Papadimitriou 02].

Theorem I.I. (Degree sequence.) Let $Z_{k}(t)$ denote the number of vertices of degree $k$, $k \geq 3$, at time $t$. For every $t \geq 1$ and $k \geq 3$, there exists a constant $b_{k}$ depending on $k$ such that

$$
\left|\mathbb{E}\left[Z_{k}(t)\right]-b_{k} t\right| \leq K, \quad \text { where } K=3.6 .
$$

Furthermore, for $t$ sufficiently large and for all $\lambda>0$,

$$
\operatorname{Pr}\left[\left|Z_{k}(t)-\mathbb{E}\left[Z_{k}(t)\right]\right| \geq \lambda\right] \leq e^{-\lambda^{2} / 72 t} .
$$


For previous weaker results on the degree sequence, see [Wu et al. 06, Zhou et al. 05]. An immediate corollary that proves the strong concentration of $Z_{k}(t)$ around its expectation is obtained from Theorem 1.1 and a union bound by setting $\lambda=10 \sqrt{t \log t}$. Specifically, we have the following corollary.

Corollary 1.2. For all possible degrees $k$,

$$
\operatorname{Pr}\left[\left|Z_{k}(t)-\mathbb{E}\left[Z_{k}(t)\right]\right| \geq 10 \sqrt{t \log t}\right]=o(1) .
$$

The next theorem provides insight into the asymptotic growth of the highest degrees of RANs and is crucial in proving Theorem 1.4.

Theorem I.3. (Highest degrees.) Let $\Delta_{1} \geq \Delta_{2} \geq \cdots \geq \Delta_{k}$ be the $k$ highest degrees of the $R A N G_{t}$ at time $t$, where $k$ is a fixed positive integer. Also, let $f(t)$ be a function such that $f(t) \rightarrow+\infty$ as $t \rightarrow+\infty$. Then with high probability (whp), ${ }^{1}$

$$
\frac{t^{1 / 2}}{f(t)} \leq \Delta_{1} \leq t^{1 / 2} f(t)
$$

and for $i=2, \ldots, k$,

$$
\Delta_{i-1}-\Delta_{i} \geq \frac{t^{1 / 2}}{f(t)}
$$

The increasing function $f(t)$ cannot be removed; see [Flaxman et al. 05]. Using Theorem 1.3 and the technique of [Mihail and Papadimitriou 02], we show how the top eigenvalues of the adjacency matrix representation of a RAN grow asymptotically as $t \rightarrow+\infty$ whp.

Theorem I.4. (Largest eigenvalues.) Let $k$ be a fixed positive integer. Also, let $\lambda_{1} \geq \lambda_{2} \geq$ $\cdots \geq \lambda_{k}$ be the $k$ largest eigenvalues of the adjacency matrix of $G_{t}$. Then whp, $\lambda_{i}=(1 \pm o(1)) \sqrt{\Delta_{i}}$.

Also, we establish the following refined upper bound for the asymptotic growth of the diameter.

\footnotetext{
${ }^{1}$ An event $A_{t}$ holds with high probability if $\lim _{t \rightarrow+\infty} \operatorname{Pr}\left[A_{t}\right]=1$.
} 
Theorem 1.5. (Diameter.) The diameter $d\left(G_{t}\right)$ of $G_{t}$ satisfies in probability $d\left(G_{t}\right) \leq$ $\rho \log t$, where $1 / \rho=\eta$ is the unique solution less than 1 of the equation $\eta-1-$ $\log \eta=\log 3$.

The outline of the paper is as follows: in Section 2, we briefly present related work and technical preliminaries needed for our analysis. We prove Theorems 1.1, 1.3, 1.4, and 1.5 in Sections 3, 4, 5, and 6 respectively. For the reader's convenience, proofs of certain lemmas are omitted from the main part of our paper and are included in an appendix, Section 8. Finally, in Section 7, we conclude by suggesting a few open problems.

\section{Related Work}

Apollonius of Perga was a Greek geometer and astronomer known for his writings on conic sections. He introduced the problem of sphere packing, whose classical solution, the so-called Apollonian packing [Graham et al. 03], exhibits a powerlaw behavior. Specifically, the circle size distribution follows a power law with exponent around 1.3 [Boyd 82]. Apollonian Networks (ANs) were introduced in [Andrade et al. 05] and independently in [Doye and Massen 05]. Random Apollonian networks (RANs) were introduced in [Zhou et al. 05]. Their degree sequence was analyzed inaccurately in that work (see comment in [Wu et al. 06]), and subsequently using a methodology from physics in [Wu et al. 06].

Eigenvalues of RANs have been studied only experimentally [Andrade and Miranda 05]. Concerning the diameters of RANs, they have been shown to grow logarithmically [Zhou et al. 05] using heuristic arguments (see, for instance, [Zhou et al. 05, equation (b.6)]).

RANs are planar 3-trees, a special case of random $k$-trees [Kloks 94], whose degree distribution of was analyzed in [Cooper and Uehara 10] and [Gao 09]. In RANs - in contrast to random $k$-trees - the random $k$ clique chosen at each step has never previously been selected. For example, in a two-dimensional RAN, each chosen face is subdivided into three new faces by connecting the incoming vertex to the vertices of the boundary.

Due to their power-law properties, random $k$-trees have been proposed as a model for complex networks; see, e.g., [Cooper and Uehara 10, Gao and Hobson 06] and references therein. Recently, a variant of $k$-trees, called ordered increasing $k$-trees, has been proposed and analyzed in [Panholzer and Seitz 10]. Closely related to RANs but not the same are random Apollonian network structures, which have been analyzed in [Bodini et al. 07, Darrasse and Soria 07, Darrasse et al. 10]. 
In [Bollobás et al. 01], the authors proved rigorously the power-law distribution of the Barabási-Albert model [Barabási and Albert 99]. Rigorous results for eigenvalue-related properties of real-world graphs using various random graph models have been proved in [Chung et al. 03, Flaxman et al. 05, Mihail and Papadimitriou 02].

In Section 3, we invoke the following useful lemma.

Lemma 2.I. [Chung Graham and Lu 06, Lemma 3.1] Suppose that a sequence $\left\{a_{t}\right\}$ satisfies the recurrence

$$
a_{t+1}=\left(1-\frac{b_{t}}{t+t_{1}}\right) a_{t}+c_{t}
$$

for $t \geq t_{0}$. Furthermore, suppose $\lim _{t \rightarrow+\infty} b_{t}=b>0$ and $\lim _{t \rightarrow+\infty} c_{t}=c$. Then $\lim _{t \rightarrow+\infty} a_{t} / t$ exists, and

$$
\lim _{t \rightarrow+\infty} \frac{a_{t}}{t}=\frac{c}{1+b}
$$

In Section 3, we also use the Azuma-Hoeffding inequality [Azuma 67, Hoeffding 63].

Lemma 2.2. (Azuma-Hoeffding inequality.) Let $\lambda>0$. Also, let $\left(X_{t}\right)_{t=0}^{n}$ be a martingale with $\left|X_{t+1}-X_{t}\right| \leq c$ for $t=0, \ldots, n-1$. Then

$$
\operatorname{Pr}\left[\left|X_{n}-X_{0}\right| \geq \lambda\right] \leq \exp \left(-\frac{\lambda^{2}}{2 c^{2} n}\right) \text {. }
$$

\section{Proof of Theorem I.I}

We decompose our proof into a sequence of lemmas. For brevity, let $N_{k}(t)=$ $\mathbb{E}\left[Z_{k}(t)\right], k \geq 3$. Also, let $d_{v}(t)$ be the degree of vertex $v$ at time $t$, and let $\mathbf{1}\left(d_{v}(t)=k\right)$ be an indicator variable that equals 1 if $d_{v}(t)=k$, and 0 otherwise. Then for every $k \geq 3$, we can express the expected number $N_{k}(t)$ of vertices of degree $k$ as a sum of expectations of indicator variables:

$$
N_{k}(t)=\sum_{v} \mathbb{E}\left[\mathbf{1}\left(d_{v}(t)=k\right)\right]
$$

We distinguish two cases in the following. 
Case 1: $k=3$. Observe that a vertex of degree 3 is created only by an insertion of a new vertex. The expectation $N_{3}(t)$ satisfies the following recurrence: ${ }^{2}$

$$
N_{3}(t+1)=N_{3}(t)+1-\frac{3 N_{3}(t)}{2 t+1} .
$$

The basis for $(3.2)$ is $N_{3}(1)=4$. We prove the following lemma, which shows that $\lim _{t \rightarrow+\infty} N_{3}(t) / t=2 / 5$.

Lemma 3.I. For all $t \geq 1, N_{3}(t)$ satisfies the following inequality:

$$
\left|N_{3}(t)-\frac{2}{5} t\right| \leq K, \quad \text { where } K=3.6 .
$$

Proof. We use induction. Assume that $N_{3}(t)=2 t / 5+e_{3}(t)$, where $e_{3}(t)$ stands for the error term. We wish to prove that for all $t,\left|e_{3}(t)\right| \leq K$. The result trivially holds for $t=1$. We also see that for $t=1$, inequality (3.3) is tight. Assume that the result holds for some $t$. We show that it holds for $t+1$ :

$$
\begin{aligned}
& N_{3}(t+1)=N_{3}(t)+1-\frac{3 N_{3}(t)}{2 t+1} \\
& \Longrightarrow e_{3}(t+1)=e_{3}(t)+\frac{3}{5}-\frac{6 t+15 e_{3}(t)}{10 t+5}=e_{3}(t)\left(1-\frac{3}{2 t+1}\right)+\frac{3}{5(2 t+1)} \\
& \Longrightarrow\left|e_{3}(t+1)\right| \leq K\left(1-\frac{3}{2 t+1}\right)+\frac{3}{5(2 t+1)} \leq K
\end{aligned}
$$

Therefore, inductively, (3.3) holds for all $t \geq 1$.

Case $2: k \geq 4$. For $k \geq 4$, the following holds:

$$
\begin{aligned}
\mathbb{E} & {\left[\mathbf{1}\left(d_{v}(t+1)=k\right)\right] } \\
& =\mathbb{E}\left[\mathbf{1}\left(d_{v}(t)=k\right)\right]\left(1-\frac{k}{2 t+1}\right)+\mathbb{E}\left[\mathbf{1}\left(d_{v}(t)=k-1\right)\right] \frac{k-1}{2 t+1} .
\end{aligned}
$$

Therefore, we can rewrite (3.1) for $k \geq 4$ as follows:

$$
N_{k}(t+1)=N_{k}(t)\left(1-\frac{k}{2 t+1}\right)+N_{k-1}(t) \frac{k-1}{2 t+1} .
$$

\footnotetext{
${ }^{2}$ The three initial vertices participate in one face fewer than their degree. However, this slight abuse leaves our main results unchanged and simplifies the exposition.
} 
Lemma 3.2. For $k \geq 3$, the limit $\lim _{t \rightarrow+\infty} N_{k}(t) / t$ exists. Specifically, let $b_{k}=$ $\lim _{t \rightarrow+\infty} N_{k}(t) / t$. Then

$$
b_{3}=\frac{2}{5}, \quad b_{4}=\frac{1}{5}, \quad b_{5}=\frac{4}{35},
$$

and for $k \geq 6$,

$$
b_{k}=\frac{24}{k(k+1)(k+2)}
$$

Furthermore, for all $k \geq 3$,

$$
\left|N_{k}(t)-b_{k} t\right| \leq K, \quad \text { where } K=3.6 .
$$

Proof. We use induction on $k$. For $k=3$, the base case, the result holds by Lemma 3.1 and specifically $b_{3}=2 / 5$. Assume that the result holds for some $k \geq 3$. We show that it holds for $k+1$, too. Rewrite (3.4) as

$$
N_{k}(t+1)=\left(1-\frac{b_{t}}{t+t_{1}}\right) N_{k}(t)+c_{t},
$$

where

$$
b_{t}=\frac{k}{2}, \quad t_{1}=\frac{1}{2}, \quad c_{t}=N_{k-1}(t) \frac{k-1}{2 t+1} .
$$

Clearly, $\lim _{t \rightarrow+\infty} b_{t}=k / 2>0$ and

$$
\lim _{t \rightarrow+\infty} c_{t}=\lim _{t \rightarrow+\infty} b_{k-1} t \frac{k-1}{2 t+1}=b_{k-1} \frac{k-1}{2} .
$$

Hence by Lemma 2.1,

$$
\lim _{t \rightarrow+\infty} \frac{N_{k}(t)}{t}=\frac{(k-1) b_{k-1} / 2}{1+k / 2}=b_{k-1} \frac{k-1}{k+2} .
$$

Since $b_{3}=2 / 5$, we obtain that $b_{4}=1 / 5, b_{5}=4 / 35$, and for every $k \geq 6$,

$$
b_{k}=\frac{24}{k(k+1)(k+2)} \text {. }
$$

This shows that the degree sequence of RANs follows a power-law distribution with exponent 3 .

Now we prove (3.5). The case $k=3$ was proved in Lemma 3.1. Let $e_{k}(t)=$ $N_{k}(t)-b_{k} t$. Assume that the result holds for some $k \geq 3$, i.e., $\left|e_{k}(t)\right| \leq K$, where $K=3.6$. We show that it holds for $k+1$, too. Substituting in (3.4) and using 
the fact that $b_{k-1}(k-1)=b_{k}(k+2)$, we obtain

$$
\begin{aligned}
e_{k}(t+1)=e_{k}(t)+ & \frac{k-1}{2 t+1} e_{k-1}(t)-\frac{k}{2 t+1} e_{k}(t) \\
\Longrightarrow\left|e_{k}(t+1)\right| & \leq\left|\left(1-\frac{k}{2 t+1}\right) e_{k}(t)\right|+\left|\frac{k-1}{2 t+1} e_{k-1}(t)\right| \leq K\left(1-\frac{1}{2 t+1}\right) \\
& \leq K .
\end{aligned}
$$

Hence by induction, (3.5) holds for all $k \geq 3$.

Finally, the following lemma provides the concentration of $Z_{k}(t)$ around its expected value for $k \geq 3$. This lemma applies Lemma 2.2 and completes the proof of Theorem 1.1 .

Lemma 3.3. Let $\lambda>0$. For $k \geq 3$,

$$
\operatorname{Pr}\left[\left|Z_{k}(t)-\mathbb{E}\left[Z_{k}(t)\right]\right| \geq \lambda\right] \leq e^{-\lambda^{2} / 72 t}
$$

Proof. Let $(\Omega, \mathcal{F}, \mathbb{P})$ be the probability space induced by the construction of a RAN after $t$ insertions. Fix $k$, where $k \geq 3$, and let $\left(X_{i}\right)_{i \in\{0,1, \ldots, t\}}$ be the martingale sequence defined by $X_{i}=\mathbb{E}\left[Z_{k}(t) \mid \mathcal{F}_{i}\right]$, where $\mathcal{F}_{0}=\{\varnothing, \Omega\}$ and $\mathcal{F}_{i}$ is the $\sigma$-algebra generated by the RAN process after $i$ steps. Observe that $X_{0}=\mathbb{E}\left[Z_{k}(t) \mid\{\varnothing, \Omega\}\right]=N_{k}(t), X_{t}=Z_{k}(t)$. We show that $\left|X_{i+1}-X_{i}\right| \leq 6$ for $i=0, \ldots, t-1$.

Let $P_{j}=\left(Y_{1}, \ldots, Y_{j-1}, Y_{j}\right), P_{j}^{\prime}=\left(Y_{1}, \ldots, Y_{j-1}, Y_{j}^{\prime}\right)$ be two sequences of face choices differing only at time $j$. Also, let $\bar{P}, \bar{P}^{\prime}$ continue from $P_{j}, P_{j}^{\prime}$ until $t$. We call the faces $Y_{j}, Y_{j}^{\prime}$ special with respect to $\bar{P}, \bar{P}^{\prime}$. We define a measure-preserving map $\bar{P} \mapsto \bar{P}^{\prime}$ in the following way: for every choice of a nonspecial face in process $\bar{P}$ at time $l$, we make the same face choice in $\bar{P}^{\prime}$ at time $l$. For every choice of a face inside the special face $Y_{j}$ in process $\bar{P}$, we make an isomorphic (with respect to, e.g., clockwise order and depth) choice of a face inside the special face $Y_{j}^{\prime}$ in process $\bar{P}^{\prime}$. Since the number of vertices of degree $k$ can change by at most 6 , i.e., the (at most) six vertices involved in the two faces $Y_{j}, Y_{j}^{\prime}$, the following holds:

$$
\left|\mathbb{E}\left[Z_{k}(t) \mid P\right]-\mathbb{E}\left[Z_{k}(t) \mid P^{\prime}\right]\right| \leq 6 .
$$

Furthermore, this holds for every $P_{j}, P_{j}^{\prime}$. We deduce that $X_{i-1}$ is a weighted mean of values, whose pairwise differences are all at most 6 .

Thus, the mean $X_{i-1}$ is at a distance of at most 6 from each of these values. Hence for every one-step refinement, we have $\left|X_{i+1}-X_{i}\right| \leq 6 \forall i \in\{0, \ldots, t-1\}$. Applying the Azuma-Hoeffding inequality as stated in Lemma 2.2, we obtain

$$
\operatorname{Pr}\left[\left|Z_{k}(t)-\mathbb{E}\left[Z_{k}(t)\right]\right| \geq \lambda\right] \leq 2 e^{-\lambda^{2} / 72 t} .
$$




\section{Proof of Theorem I.3}

We decompose the proof of Theorem 1.3 into several lemmas. Specifically, the proof follows directly from Lemmas 4.2, 4.4-4.7. We partition the vertices into three sets: those added before $t_{0}$, between $t_{0}$ and $t_{1}$, and after $t_{1}$, where $t_{0}=\log \log \log (f(t))$ and $t_{1}=\log \log (f(t))$. Recall that $f(t)$ is a function such that $\lim _{t \rightarrow+\infty} f(t)=+\infty$. We define a supernode to be a collection of vertices, and the degree of the supernode to be the sum of the degrees of its vertices.

Lemma 4.I. Let $d_{t}(s)$ denote the degree of vertex $s$ at time $t$, and let $a^{(k)}=a(a+$ $1) \cdots(a+k-1)$ denote the rising factorial function. Then for every positive integer $k$,

$$
\mathbb{E}\left[d_{t}(s)^{(k)}\right] \leq \frac{(k+2) !}{2}\left(\frac{2 t}{s}\right)^{k / 2} .
$$

Proof. As we mentioned in the proof of Theorem 1.1, the three initial vertices 1,2,3 have one face fewer than their degree, whereas all other vertices have degree equal to the number of faces surrounding them. In this proof, we treat both cases, but we omit this distinction in all other proofs.

Case 1: $s \geq 4$. Note that $d_{s}(s)=3$. By conditioning successively, we obtain

$$
\begin{aligned}
\mathbb{E} & {\left[d_{t}(s)^{(k)}\right]=\mathbb{E}\left[\mathbb{E}\left[d_{t}(s)^{(k)} \mid d_{t-1}(s)\right]\right] } \\
& =\mathbb{E}\left[\left(d_{t-1}(s)\right)^{(k)}\left(1-\frac{d_{t-1}(s)}{2 t-1}\right)+\left(d_{t-1}(s)+1\right)^{(k)} \frac{d_{t-1}(s)}{2 t-1}\right] \\
& =\mathbb{E}\left[\left(d_{t-1}(s)\right)^{(k)}\left(1-\frac{d_{t-1}(s)}{2 t-1}\right)+\left(d_{t-1}(s)\right)^{(k)} \frac{d_{t-1}(s)+k}{d_{t-1}(s)} \frac{d_{t-1}(s)}{2 t-1}\right] \\
& =\mathbb{E}\left[\left(d_{t-1}(s)\right)^{(k)}\right]\left(1+\frac{k}{2 t-1}\right)=\cdots=3^{(k)} \prod_{t^{\prime}=s+1}^{t}\left(1+\frac{k}{2 t^{\prime}-1}\right) \\
& \leq 3^{(k)} \exp \left(\sum_{t^{\prime}=s+1}^{t} \frac{k}{2 t^{\prime}-1}\right) \leq 3^{(k)} \exp \left(k \int_{s}^{t} \frac{\mathrm{d} x}{2 x-1}\right) \\
& \leq \frac{(k+2) !}{2} \exp \left(\frac{k}{2} \log \frac{t-1 / 2}{s-1 / 2}\right) \leq \frac{(k+2) !}{2}\left(\frac{2 t}{s}\right)^{k / 2} .
\end{aligned}
$$


Case 2: $s \in\{1,2,3\}$. Note that initially, the degree of any such vertex is 2. For $k \geq 0$, we have

$$
\begin{aligned}
& \mathbb{E}\left[d_{t}(s)^{(k)}\right]=\mathbb{E}\left[\mathbb{E}\left[d_{t}(s)^{(k)} \mid d_{t-1}(s)\right]\right] \\
& =\mathbb{E}\left[\left(d_{t-1}(s)\right)^{(k)}\left(1-\frac{d_{t-1}(s)-1}{2 t-1}\right)+\left(d_{t-1}(s)+1\right)^{(k)} \frac{d_{t-1}(s)-1}{2 t-1}\right] \\
& =\mathbb{E}\left[\left(d_{t-1}(s)\right)^{(k)}\left(1+\frac{k}{2 t-1}\right)-\left(d_{t-1}(s)\right)^{(k)} \frac{k}{(2 t-1) d_{t-1}(s)}\right] \\
& \quad \leq \mathbb{E}\left[\left(d_{t-1}(s)\right)^{(k)}\right]\left(1+\frac{k}{2 t-1}\right) \leq \cdots \leq \frac{(k+2) !}{2}\left(\frac{2 t}{s}\right)^{k / 2} .
\end{aligned}
$$

Lemma 4.2. The degree $X_{t}$ of the supernode $V_{t_{0}}$ of vertices added before time $t_{0}$ is at least $t_{0}^{1 / 4} \sqrt{t}$ whp.

Proof. We consider a modified process $\mathcal{Y}$ coupled with the RAN process. Figure 2 illustrates this coupling. Specifically, let $Y_{t}$ be the modified degree of the supernode in the modified process $\mathcal{Y}$, which is defined as follows: for any type of insertion in the original RAN process (note that there exist three types of insertions with respect to how the degree $X_{t}$ of the supernode (black circle) is affected; see also Figure 2), $Y_{t}$ increases by 1 . We also define $X_{t_{0}}=Y_{t_{0}}$. Note that $X_{t} \geq Y_{t}$ for all $t \geq t_{0}$. Let

$$
d_{0}=X_{t_{0}}=Y_{t_{0}}=6 t_{0}+6 \quad \text { and } \quad p_{r}^{*}=p^{*}=\operatorname{Pr}\left[Y_{t}=d_{0}+r \mid Y_{t_{0}}=d_{0}\right] .
$$

The following technical claim is proved in Section 8 .

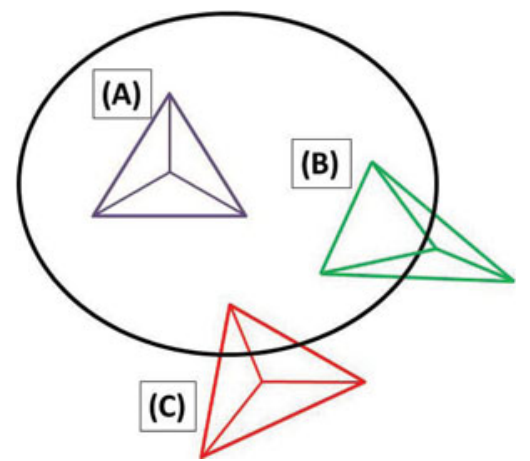

Figure 2. Coupling used in Lemma 4.2. 
Claim 4.3. We have

$$
p^{*} \leq\left(\begin{array}{c}
d_{0}+r-1 \\
d_{0}-1
\end{array}\right)\left(\frac{2 t_{0}+3}{2 t+1}\right)^{d_{0} / 2} e^{3 / 2+t_{0}-d_{0} / 2+2 r / 3 \sqrt{t}} .
$$

Let $\mathcal{A}_{1}$ denote the event that the supernode consisting of the first $t_{0}$ vertices has degree $Y_{t}$ in the modified process $\mathcal{Y}$ less than $t_{0}^{1 / 4} \sqrt{t}$. Note that since

$$
\left\{X_{t} \leq t_{0}^{1 / 4} \sqrt{t}\right\} \subseteq\left\{Y_{t} \leq t_{0}^{1 / 4} \sqrt{t}\right\}
$$

it suffices to prove that $\operatorname{Pr}\left[Y_{t} \leq t_{0}^{1 / 4} \sqrt{t}\right]=o(1)$. Using Claim 4.3, we obtain

$$
\begin{aligned}
\operatorname{Pr}\left[\mathcal{A}_{1}\right] & \leq \sum_{r=0}^{t_{0}^{1 / 4} \sqrt{t}-\left(6 t_{0}+6\right)}\left(\begin{array}{c}
r+6 t_{0}+5 \\
6 t_{0}+5
\end{array}\right)\left(\frac{2 t_{0}+3}{2 t+1}\right)^{3 t_{0}+3} e^{-3 / 2-2 t_{0}+2 t_{0}^{1 / 4} / 3} \\
& \leq t_{0}^{1 / 4} t^{1 / 2} \frac{\left(t_{0}^{1 / 4} t^{1 / 2}\right)^{6 t_{0}+5}}{\left(6 t_{0}+5\right) !}\left(\frac{2 t_{0}+3}{2 t+1}\right)^{3 t_{0}+3} e^{-3 / 2-2 t_{0}+2 t_{0}^{1 / 4} / 3} \\
& \leq\left(\frac{t}{2 t+1}\right)^{3 t_{0}+3} \frac{t_{0}^{3 t_{0} / 2+3 / 2}\left(2 t_{0}+3\right)^{3 t_{0}+3}}{\left(6 t_{0}+5\right)^{6 t_{0}+5}} e^{4 t_{0}+7 / 2+2 / 3 t_{0}^{1 / 4}} \\
& \leq 2^{-\left(3 t_{0}+3\right)} \frac{e^{4 t_{0}+7 / 2+2 / 3 t_{0}^{1 / 4}}}{\left(6 t_{0}+5\right)^{3 / 2 t_{0}+1 / 2}}=o(1) .
\end{aligned}
$$

Lemma 4.4. No vertex added after $t_{1}$ has degree exceeding $t_{0}^{-2} t^{1 / 2}$ whp.

Proof. Let $\mathcal{A}_{2}$ denote the event that some vertex added after $t_{1}$ has degree exceeding $t_{0}^{-2} t^{1 / 2}$. We use a union bound, a third-moment argument, and Lemma 4.1 to prove that $\operatorname{Pr}\left[\mathcal{A}_{2}\right]=o(1)$. Specifically,

$$
\begin{aligned}
\operatorname{Pr}\left[\mathcal{A}_{2}\right] & \leq \sum_{s=t_{1}}^{t} \operatorname{Pr}\left[d_{t}(s) \geq t_{0}^{-2} t^{1 / 2}\right]=\sum_{s=t_{1}}^{t} \operatorname{Pr}\left[d_{t}(s)^{(3)} \geq\left(t_{0}^{-2} t^{1 / 2}\right)^{(3)}\right] \\
& \leq t_{0}^{6} t^{-3 / 2} \sum_{s=t_{1}}^{t} \mathbb{E}\left[d_{t}(s)^{(3)}\right] \leq 5 ! \sqrt{2} t_{0}^{6} \sum_{s=t_{1}}^{t} s^{-3 / 2} \leq 5 ! 2 \sqrt{2} t_{0}^{6} t_{1}^{-1 / 2}=o(1) .
\end{aligned}
$$

Lemma 4.5. No vertex added before $t_{1}$ has degree exceeding $t_{0}^{1 / 6} t^{1 / 2}$ whp.

Proof. Let $\mathcal{A}_{3}$ denote the event that some vertex added before $t_{1}$ has degree exceeding $t_{0}^{1 / 6} t^{1 / 2}$. We use again a third-moment argument and Lemma 4.1 to prove 
that $\operatorname{Pr}\left[\mathcal{A}_{3}\right]=o(1)$ :

$$
\begin{aligned}
\operatorname{Pr}\left[\mathcal{A}_{3}\right] & \leq \sum_{s=1}^{t_{1}} \operatorname{Pr}\left[d_{t}(s) \geq t_{0}^{1 / 6} t^{1 / 2}\right]=\sum_{s=1}^{t_{1}} \operatorname{Pr}\left[d_{t}(s)^{(3)} \geq\left(t_{0}^{1 / 6} t^{1 / 2}\right)^{(3)}\right] \\
& \leq t_{0}^{-1 / 2} t^{-3 / 2} \sum_{s=1}^{t_{1}} \mathbb{E}\left[d_{t}(s)^{(3)}\right] \leq t_{0}^{-1 / 2} t^{-3 / 2} \sum_{s=1}^{t_{1}} 5 ! \sqrt{2} \frac{t^{3 / 2}}{s^{3 / 2}} \\
& \leq 5 ! \sqrt{2} \zeta(3 / 2) t_{0}^{-1 / 2}=o(1)
\end{aligned}
$$

where $\zeta(3 / 2)=\sum_{s=1}^{+\infty} s^{-3 / 2} \approx 2.612$.

Lemma 4.6. The $k$ highest degrees are added before $t_{1}$ and have degree $\Delta_{i}$ bounded by $t_{0}^{-1} t^{1 / 2} \leq \Delta_{i} \leq t_{0}^{1 / 6} t^{1 / 2}$ whp, $i=1, \ldots, k$.

Proof. For the upper bound, it suffices to show that $\Delta_{1} \leq t_{0}^{1 / 6} t^{1 / 2}$. This follows immediately by Lemmas 4.4 and 4.5. The lower bound follows directly from Lemmas 4.2, 4.4, and 4.5. Assume that at most $k-1$ vertices added before $t_{1}$ have degree exceeding the lower bound $t_{0}^{-1} t^{1 / 2}$. Then the total degree of the supernode formed by the first $t_{0}$ vertices is $O\left(t_{0}^{1 / 6} \sqrt{t}\right)$. This contradicts Lemma 4.2.

Finally, since each vertex $s \geq t_{1}$ has degree at most $t_{0}^{-2} \sqrt{t} \ll t_{0}^{-1} t^{1 / 2}$, the $k$ highest-degree vertices are added before $t_{1}$ whp.

The proof of Theorem 1.3 is completed with the following lemma, whose proof is deferred to Section 8 .

Lemma 4.7. The $k$ highest degrees satisfy $\Delta_{i} \leq \Delta_{i-1}-\sqrt{t} / f(t)$ whp, $i=1, \ldots, k$.

\section{Proof of Theorem I.4}

Having computed the highest degrees of a RAN in Section 4, eigenvalues are computed by adapting existing techniques [Chung et al. 03, Flaxman et al. 05, Mihail and Papadimitriou 02]. We decompose the proof of Theorem 1.4 into Lemmas 5.1-5.4. Specifically, in Lemmas 5.1 and 5.2, we bound the degrees and codegrees (the codegree of vertices $i, j$ is the number of their common neighbors). Having these bounds, we decompose the graph into a star forest and show in Lemmas 5.3 and 5.4 that its largest eigenvalues, which are $(1 \pm o(1)) \sqrt{\Delta_{i}}$, dominate the eigenvalues of the remaining graph. This technique was pioneered in [Mihail and Papadimitriou 02]. 
We partition the vertices into three sets $S_{1}, S_{2}, S_{3}$. Specifically, let $S_{i}$ be the set of vertices added after time $t_{i-1}$ and at or before time $t_{i}$, where

$$
t_{0}=0, \quad t_{1}=t^{1 / 8}, \quad t_{2}=t^{9 / 16}, \quad t_{3}=t .
$$

Lemma 5.I. For every $\epsilon>0$ and $f(t)$ with $f(t) \rightarrow+\infty$ as $t \rightarrow+\infty$, the following holds whp: for all $s$ with $f(t) \leq s \leq t$, for all vertices $r \leq s$, we have $d_{s}(r) \leq$ $s^{1 / 2+\epsilon} r^{-1 / 2}$.

Proof. Set $q=\lceil 4 / \epsilon\rceil$. We use Lemma 4.1, a union bound, and Markov's inequality to obtain

$$
\begin{aligned}
\operatorname{Pr} & {\left[\bigcup_{s=f(t)}^{t} \bigcup_{r=1}^{s}\left\{d_{s}(r) \geq s^{1 / 2+\epsilon} r^{-1 / 2}\right\}\right] } \\
& \leq \sum_{s=f(t)}^{t} \sum_{r=1}^{s} \operatorname{Pr}\left[d_{s}(r)^{(q)} \geq\left(s^{1 / 2+\epsilon} r^{-1 / 2}\right)^{(q)}\right] \\
& \leq \sum_{s=f(t)}^{t} \sum_{r=1}^{s} \operatorname{Pr}\left[d_{s}(r)^{(q)} \geq\left(s^{-(q / 2+q \epsilon)} r^{q / 2}\right)\right] \\
& \leq \sum_{s=f(t)}^{t} \sum_{r=1}^{s} \frac{(q+2) !}{2}\left(\frac{2 s}{r}\right)^{q / 2} s^{-q / 2} s^{-q \epsilon} r^{q / 2}=\frac{(q+2) !}{2} 2^{q / 2} \sum_{s=f(t)}^{t} s^{1-q \epsilon} \\
& \leq \frac{(q+2) !}{2} 2^{q / 2} \int_{f(t)-1}^{t} x^{1-q \epsilon} \mathrm{d} x \leq \frac{(q+2) !}{2(q \epsilon-2)} 2^{q / 2}(f(t)-1)^{2-q \epsilon}=o(1) .
\end{aligned}
$$

Lemma 5.2. Let $S_{3}^{\prime}$ be the set of vertices in $S_{3}$ that are adjacent to more than one vertex of $S_{1}$. Then $\left|S_{3}^{\prime}\right| \leq t^{1 / 6}$ whp.

Proof. First, observe that when vertex $s$ is inserted, it becomes adjacent to more than one vertex of $S_{1}$ if the face chosen by $s$ has at least two vertices in $S_{1}$. We call the latter property $\mathcal{A}$, and we write $s \in \mathcal{A}$ when $s$ satisfies it. At time $t_{1}$, there exist $2 t_{1}+1$ faces altogether, which consist of faces whose three vertices are all from $S_{1}$. At time $s \geq t_{2}$, there can be at most $6 t_{1}+3$ faces with at least two vertices in $S_{1}$, since each of the original $2 t_{1}+1$ faces can give rise to at most three new faces with at least two vertices in $s_{1}$. Consider a vertex $s \in S_{3}$, i.e., $s \geq t_{2}$. By the above argument,

$$
\operatorname{Pr}\left[\left|N(s) \cap S_{1}\right| \geq 2\right] \leq \frac{6 t_{1}+3}{2 t+1} .
$$


Writing $\left|S_{3}^{\prime}\right|$ as a sum of indicator variables, i.e., $\left|S_{3}^{\prime}\right|=\sum_{s=t_{2}}^{t} I(s \in \mathcal{A})$, and taking the expectation, we obtain

$$
\begin{aligned}
\mathbb{E}\left[\left|S_{3}^{\prime}\right|\right] & \leq \sum_{s=t_{2}}^{t} \frac{6 t_{1}+3}{2 t+1} \leq\left(6 t_{1}+3\right) \int_{t_{2}}^{t}(2 x+1)^{-1} \mathrm{~d} x \\
& \leq\left(3 t^{1 / 8}+\frac{3}{2}\right) \ln \frac{2 t+1}{2 t_{2}+1}=o\left(t^{1 / 7}\right) .
\end{aligned}
$$

By Markov's inequality:

$$
\operatorname{Pr}\left[\left|S_{3}^{\prime}\right| \geq t^{1 / 6}\right] \leq \frac{\mathbb{E}\left[\left|S_{3}^{\prime}\right|\right]}{t^{1 / 6}}=o(1) .
$$

Therefore, we conclude that $\left|S_{3}^{\prime}\right| \leq t^{1 / 6}$ whp.

Lemma 5.3. Let $F \subseteq G$ be the star forest consisting of edges between $S_{1}$ and $S_{3}-S_{3}^{\prime}$. Let $\Delta_{1} \geq \Delta_{2} \geq \cdots \geq \Delta_{k}$ denote the $k$ highest degrees of $G$. Then $\lambda_{i}(F)=(1-$ $o(1)) \sqrt{\Delta_{i}}$ whp.

Proof. It suffices to show that $\Delta_{i}(F)=(1-o(1)) \Delta_{i}(G)$ for $i=1, \ldots, k$. Note that since the $k$ highest vertices are inserted before $t_{1}$ whp, the edges they lose are the edges between $S_{1}$ and those incident to $S_{3}^{\prime}$ and $S_{2}$, and we know how to bound the cardinalities of all these sets. Specifically, by Lemma $5.2,\left|S_{3}^{\prime}\right| \leq t^{1 / 6} \mathrm{whp}$, and by Theorem 1.3, the maximum degrees in $G_{t_{1}}, G_{t_{2}}$ are less than $t^{1 / 8}, t^{5 / 16}$, respectively, whp. Also, by Theorem $1.3, \Delta_{i}(G) \geq \sqrt{t} / \log t$. Hence, we obtain

$$
\Delta_{i}(F) \geq \Delta_{i}(G)-t^{1 / 8}-t^{5 / 16}-t^{1 / 6}=(1-o(1)) \Delta_{i}(G) .
$$

To complete the proof of Theorem 1.4, it suffices to prove that $\lambda_{1}(H)$ is $o\left(\lambda_{k}(F)\right)$, where $H=G-F$. We prove this in the following lemma. The proof is based on bounding the maximum degree of appropriately defined subgraphs using Lemma 5.1 and standard inequalities from spectral graph theory [Chung Graham 97, Flaxman et al. 05, Mihail and Papadimitriou 02].

Lemma 5.4. $\lambda_{1}(H)=o\left(t^{1 / 4}\right) w h p$.

Proof. Gershgorin's theorem states that the maximum eigenvalue of every graph is bounded by the maximum degree [Strang 05]. We bound the eigenvalues of $H$ by bounding the maximum eigenvalues of six different induced subgraphs. Specifically, let $H_{i}=H\left[S_{i}\right], H_{i j}=H\left(S_{i}, S_{j}\right)$, where $H[S]$ is the subgraph induced by the vertex set $S$ and $H(S, T)$ is the subgraph containing only edges with one 
vertex is $S$ and the other in $T$. We use Lemma 5.3 to bound $\lambda_{1}\left(H\left(S_{1}, S_{3}\right)\right)$ and Lemma 5.2 for the other eigenvalues. We set $\epsilon=1 / 64$ :

$$
\begin{aligned}
& \lambda_{1}\left(H_{1}\right) \leq \Delta_{1}\left(H_{1}\right) \leq t_{1}^{1 / 2+\epsilon}=t^{33 / 512}, \\
& \lambda_{1}\left(H_{2}\right) \leq \Delta_{1}\left(H_{2}\right) \leq t_{2}^{1 / 2+\epsilon} t_{1}^{-1 / 2}=t^{233 / 1024}, \\
& \lambda_{1}\left(H_{3}\right) \leq \Delta_{1}\left(H_{3}\right) \leq t_{3}^{1 / 2+\epsilon} t_{2}^{-1 / 2}=t^{15 / 64}, \\
& \lambda_{1}\left(H_{12}\right) \leq \Delta_{1}\left(H_{12}\right) \leq t_{2}^{1 / 2+\epsilon}=t^{297 / 1024} \\
& \lambda_{1}\left(H_{23}\right) \leq \Delta_{1}\left(H_{23}\right) \leq t_{3}^{1 / 2+\epsilon} t_{1}^{-1 / 2}=t^{29 / 64} \\
& \lambda_{1}\left(H_{13}\right) \leq \Delta_{1}\left(H_{13}\right) \leq t^{1 / 6}
\end{aligned}
$$

Therefore, by a union bound we obtain whp

$$
\lambda_{1}(H) \leq \sum_{i=1}^{3} \lambda_{1}\left(H_{i}\right)+\sum_{i<j} \lambda_{1}\left(H_{i j}\right)=o\left(t^{1 / 4}\right) .
$$

\section{Proof of Theorem 1.5}

Before we give the proof of Theorem 1.5, we give a simple proof that the diameter of a RAN is $O(\log t)$ whp. We begin with a necessary definition for the proof of Claim 6.1 below. We define the depth of a face recursively. Initially, we have three faces, whose depth equals 1 ; see Figure 1(a). For each new face $\beta$ created by subdividing a face $\gamma$, we have $\operatorname{depth}(\beta)=\operatorname{depth}(\gamma)+1$. An example is shown in Figure 3, where each face is labeled with its corresponding depth.

Claim 6.I. The diameter $d\left(G_{t}\right)$ satisfies $d\left(G_{t}\right)=O(\log t)$ whp.

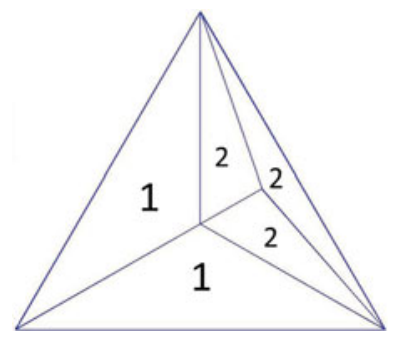

Figure 3. An instance of the process for $t=2$. Each face is labeled with its depth. 
Proof. A simple but key observation is that if $k^{*}$ is the maximum depth of a face, then $d\left(G_{t}\right)=O\left(k^{*}\right)$. Hence, we need to bound from above the depth of a given face after $t$ rounds. Let $F_{t}(k)$ be the number of faces of depth $k$ at time $t$. Then

$$
\begin{aligned}
\mathbb{E}\left[F_{t}(k)\right] & =\sum_{1 \leq t_{1}<\cdots<t_{k} \leq t} \prod_{j=1}^{k} \frac{1}{2 t_{j}+1} \leq \frac{1}{k !}\left(\sum_{j=1}^{t} \frac{1}{2 j+1}\right)^{k} \leq \frac{1}{k !}\left(\frac{1}{2} \log t\right)^{k} \\
& \leq\left(\frac{e \log t}{2 k}\right)^{k+1}
\end{aligned}
$$

By the first moment method, we obtain $k^{*}=O(\log t)$ whp, and by our observation, $d\left(G_{t}\right)=O(\log t)$ whp.

The depth of a face can be formalized via a bijection between random ternary trees and RANs. Using this bijection, we prove Theorem 1.5, which gives a refined upper bound on the asymptotic growth of the diameter.

Proof of Theorem I.5. Consider the random process that starts with a single vertex tree and at every step, picks a random leaf and adds three children to it. Let $T$ be the resulting tree after $t$ steps. There exists a natural bijection between the RAN process and this process; see [Darrasse and Soria 07] and also Figure 4. The depth of $T$ in probability is $(\rho / 2) \log t$, where $1 / \rho=\eta$ is the unique solution less than 1 of the equation $\eta-1-\log \eta=\log 3$; see [Broutin and Devroye 06,

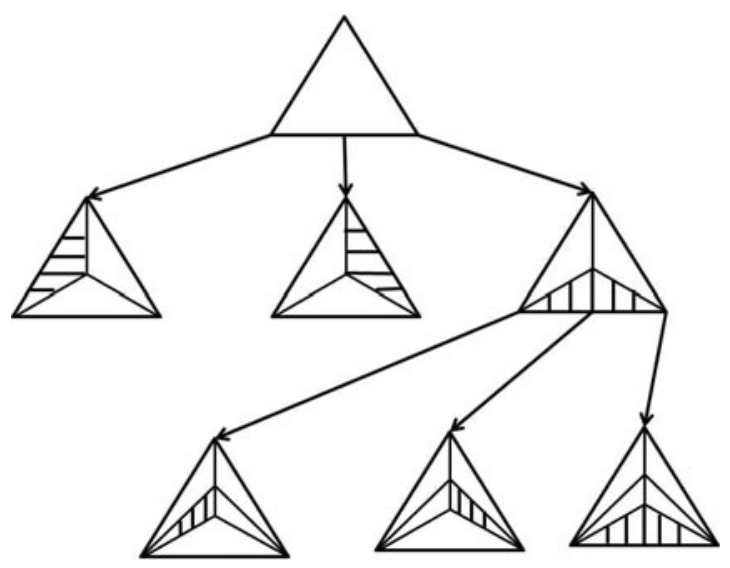

Figure 4. RANs as random ternary trees. 

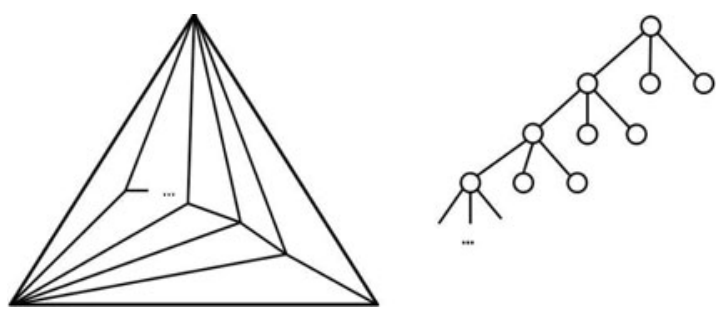

Figure 5. The height of the random ternary tree cannot be used to bound the diameter from below. The height of the random ternary tree in the figure can be arbitrarily large, but the diameter is 2 .

pp. 284-285]. ${ }^{3}$ Note that the diameter $d\left(G_{t}\right)$ is at most twice the height of the tree, and the result follows.

The above observation, i.e., the bijection between RANs and random ternary trees, cannot be used to bound the diameter from below. A counterexample is shown in Figure 5, where the height of the random ternary tree can be made arbitrarily large, but the diameter is 2 . It was proved in [Albenque and Marckert 08] that if $v, u$ are two i.i.d. uniformly random internal vertices, i.e., $v, u \geq 4$, then the distance $d(u, v)$ tends to $6 \log n / 11$ with probability 1 as the number of vertices $n$ of the RAN grows to infinity. However, to the best of our knowledge, an exact expression for the asymptotic growth of the diameter remains an open problem. Finally, it is worth mentioning that the diameter of the RAN grows faster asymptotically than the diameter of the classical preferential attachment model [Barabási and Albert 99], which whp grows like $\log t / \log \log t$; see [Bollobás and Riordan 04].

\section{Open Problems}

We propose three open problems for future work. The first concerns the diameter. Specifically, as we mentioned earlier, an interesting problem is to find an exact asymptotic expression for the diameter of a RAN. Since the time of our original writeup, it was proved in [Ebrahimzadeh et al. 13] that the diameter asymptotically is $c \log t$, where $c \approx 1.668$.

\footnotetext{
${ }^{3}$ There is a typo in [Broutin and Devroye 06]: in " $\rho$ is the unique solution greater than 1 of ..." one should replace "greater than" with "less than," based on the authors' Theorem 1. We are grateful to Abbas Mehrabian for pointing this out.
} 


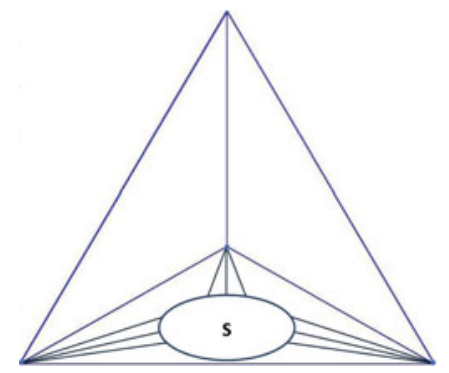

Figure 6. By the pigeonhole principle, one of the three initial faces receives $\Theta(t)$ vertices. Using Theorem 1.3, it is not hard to see that the encircled set of vertices $S$ has conductance $\phi(S) \approx \sqrt{t} / t=1 / \sqrt{t}$ whp.

Conjecture 7.I. (Conductance.) We conjecture that the conductance [Bollobás 98] of a $R A N$ is $\Theta(1 / \sqrt{t})$ whp. Figure 6 shows that $\Phi\left(G_{t}\right) \leq 1 / \sqrt{t}$.

Conjecture 7.2. (Hamiltonicity and Longest Path.) We conjecture that whp, a RAN is not Hamiltonian, but the length of the longest path is $\Omega(n)$.

Since the time of our original writeup, this conjecture has been refuted. Specifically, it is proved in [Ebrahimzadeh et al. 13] that every path has length $o(n)$. Furthermore, the authors prove that a RAN always has a path of length $(2 n-5)^{\log 2 / \log 3}$ and that the expected length of its longest path is $\Omega\left(n^{0.88}\right)$.

\section{Appendix}

In this appendix, we prove Claim 4.3 and Lemma 4.7 .

Claim 4.3. We have

$$
p^{*} \leq\left(\begin{array}{c}
d_{0}+r-1 \\
d_{0}-1
\end{array}\right)\left(\frac{2 t_{0}+3}{2 t+1}\right)^{d_{0} / 2} e^{3 / 2+t_{0}-d_{0} / 2+2 r / 3 \sqrt{t}} .
$$

Proof. Let

$$
\tau=(t_{0} \equiv \tau_{0}, \underbrace{\tau_{1}, \ldots, \tau_{r}}_{\text {insertion times }}, \tau_{r+1} \equiv t)
$$

be a vector denoting that $Y_{t}$ increases by 1 at $\tau_{i}$ for $i=1, \ldots, r$. We bound the probability $p_{\tau}$ of this event from above in the following. Note that we consider 
the case in which the vertices have the same degree as the number of faces around them. As we mentioned earlier, the other case is analyzed in exactly the same way, modulo a negligible error term:

$$
\begin{aligned}
p_{\tau}= & {\left[\prod_{k=1}^{r} \frac{d_{0}+k-1}{2 \tau_{k}+1}\right]\left[\prod_{k=0}^{r} \prod_{j=\tau_{k}+1}^{\tau_{k+1}-1}\left(1-\frac{d_{0}+k}{2 j+1}\right)\right] } \\
\leq & d_{0}\left(d_{0}+1\right) \cdots\left(d_{0}+r-1\right)\left[\prod_{k=1}^{r} \frac{1}{2 \tau_{k}+1}\right] \\
& \times \exp \left(\sum_{k=0}^{r} \sum_{j=\tau_{k}+1}^{\tau_{k+1}-1} \log \left(1-\frac{d_{0}+k}{2 j+1}\right)\right) \\
= & \frac{\left(d_{0}+r-1\right) !}{\left(d_{0}-1\right) !}\left[\prod_{k=1}^{r} \frac{1}{2 \tau_{k}+1}\right] \exp \left(\sum_{k=0}^{r} \sum_{j=\tau_{k}+1}^{\tau_{k+1}-1} \log \left(1-\frac{d_{0}+k}{2 j+1}\right)\right) .
\end{aligned}
$$

Consider now the inner sum, which we bound from above using an integral:

$$
\begin{aligned}
& \sum_{j=\tau_{k}+1}^{\tau_{k+1}-1} \log \left(1-\frac{d_{0}+k}{2 j+1}\right) \leq \int_{\tau_{k}+1}^{\tau_{k+1}} \log \left(1-\frac{d_{0}+k}{2 x+1}\right) \mathrm{d} x \\
& \leq-\left(\tau_{k+1}+\frac{1}{2}\right) \log \left(2 \tau_{k+1}+1\right) \\
& \quad+\frac{2 \tau_{k+1}+1-\left(d_{0}+k\right)}{2} \log \left(2 \tau_{k+1}+1-\left(d_{0}+k\right)\right) \\
& \quad+\left(\tau_{k}+\frac{3}{2}\right) \log \left(2 \tau_{k}+3\right)-\frac{2 \tau_{k}+3-\left(d_{0}+k\right)}{2} \log \left(2 \tau_{k}+3-\left(d_{0}+k\right)\right),
\end{aligned}
$$

since

$$
\begin{aligned}
& \int \log \left(1-\frac{d_{0}+k}{2 x+1}\right) \\
& \quad=-\left(x+\frac{1}{2}\right) \log (2 x+1)+\frac{2 x+1-\left(d_{0}+k\right)}{2} \log \left(2 x+1-\left(d_{0}+k\right)\right) .
\end{aligned}
$$

Hence we obtain

$$
\sum_{k=0}^{r} \sum_{j=\tau_{k}+1}^{\tau_{k+1}-1} \log \left(1-\frac{d_{0}+k}{2 j+1}\right) \leq A+\sum_{k=1}^{r} B_{k},
$$


where

$$
\begin{aligned}
A= & \left(\tau_{0}+\frac{3}{2}\right) \log \left(2 \tau_{0}+3\right)-\frac{2 \tau_{0}+3-d_{0}}{2} \log \left(2 \tau_{0}+3-d_{0}\right) \\
& -\left(\tau_{r+1}+\frac{1}{2}\right) \log \left(2 \tau_{r+1}+1\right) \\
& +\frac{2 \tau_{r+1}+1-\left(d_{0}+r\right)}{2} \log \left(2 \tau_{r+1}+1-\left(d_{0}+r\right)\right)
\end{aligned}
$$

and

$$
\begin{aligned}
B_{k}= & \left(\tau_{k}+\frac{3}{2}\right) \log \left(2 \tau_{k}+3\right)-\frac{2 \tau_{k}+3-\left(d_{0}+k\right)}{2} \log \left(2 \tau_{k}+3-\left(d_{0}+k\right)\right) \\
& -\left(\tau_{k}+\frac{1}{2}\right) \log \left(2 \tau_{k}+1\right) \\
& +\frac{2 \tau_{k}+1-\left(d_{0}+k-1\right)}{2} \log \left(2 \tau_{k}+1-\left(d_{0}+k-1\right)\right) .
\end{aligned}
$$

We first bound from above the quantities $B_{k}$ for $k=1, \ldots, r$. Rearranging terms and using the identity $\log (1+x) \leq x$, we obtain

$$
\begin{aligned}
B_{k}= & \left(\tau_{k}+\frac{1}{2}\right) \log \left(1+\frac{1}{\tau_{k}+\frac{1}{2}}\right)+\log \left(2 \tau_{k}+3\right) \\
& -\frac{1}{2} \log \left(2 \tau_{k}+3-\left(d_{0}+k\right)\right) \\
& -\frac{2 \tau_{k}+2-\left(d_{0}+k\right)}{2} \log \left(1+\frac{1}{2 \tau_{k}+2-\left(d_{0}+k\right)}\right) . \\
\leq & \frac{1}{2}+\frac{1}{2} \log \left(2 \tau_{k}+3\right)-\frac{1}{2} \log \left(1-\frac{d_{0}+k}{2 \tau_{k}+3}\right)
\end{aligned}
$$

First, we rearrange terms, and then we bound the term $e^{A}$ using the inequality $\exp \left(-x-x^{2} / 2\right) \geq 1-x$, which is valid for $0<x<1$ :

$$
\begin{aligned}
A= & -\left(\tau_{0}+\frac{3}{2}\right) \log \left(1-\frac{d_{0}}{2 \tau_{0}+3}\right)+\left(\tau_{r+1}+\frac{1}{2}\right) \log \left(1-\frac{d_{0}+r}{2 \tau_{r+1}+1}\right) \\
& +\frac{d_{0}}{2} \log \left(2 \tau_{0}+3-d_{0}\right) \\
& -\frac{d_{0}+r}{2} \log \left(2 \tau_{r+1}+1-\left(d_{0}+r\right)\right),
\end{aligned}
$$


which implies

$$
\begin{aligned}
e^{A}= & \left(1-\frac{d_{0}}{2 \tau_{0}+3}\right)^{-\left(\tau_{0}+3 / 2\right)}\left(1-\frac{d_{0}+r}{2 \tau_{r+1}+1}\right)^{\tau_{r+1}+\frac{1}{2}}\left(2 \tau_{0}+3-d_{0}\right)^{d_{0} / 2} \\
& \times\left(2 \tau_{r+1}+1-\left(d_{0}+r\right)\right)^{-\left(d_{0}+r\right) / 2} \\
= & \left(\frac{2 \tau_{0}+3}{2 \tau_{r+1}+1}\right)^{d_{0} / 2}\left(2 \tau_{r+1}+1\right)^{-r / 2}\left(1-\frac{d_{0}}{2 \tau_{0}+3}\right)^{-\left(\tau_{0}+3 / 2\right)+d_{0} / 2} \\
& \times\left(1-\frac{d_{0}+r}{2 \tau_{r+1}+1}\right)^{\tau_{r+1}+1 / 2-\left(d_{0}+r\right) / 2} \\
\leq & \left(\frac{2 t_{0}+3}{2 t+1}\right)^{d_{0} / 2}(2 t+1)^{-r / 2}\left(1-\frac{d_{0}}{2 \tau_{0}+3}\right)^{-\left(\tau_{0}+3 / 2\right)+d_{0} / 2} \\
& \left.\times \exp \left(-\frac{d_{0}+r}{2 t+1}-\frac{1}{2}\left(-\frac{d_{0}+r}{2 t+1}\right)^{2}\right)\left(t+\frac{1}{2}-\frac{d_{0}+r}{2}\right)\right) \\
= & \left(\frac{2 t_{0}+3}{2 t+1}\right)^{d_{0} / 2}(2 t+1)^{-r / 2}\left(1-\frac{d_{0}}{2 \tau_{0}+3}\right)^{-\left(\tau_{0}+3 / 2\right)+d_{0} / 2} \\
& \times \exp \left(-\frac{d_{0}+r}{2}+\frac{\left(d_{0}+r\right)^{2}}{8 t+4}+\frac{\left(d_{0}+r\right)^{3}}{4(2 t+1)^{2}}\right) .
\end{aligned}
$$

Now we bound from above the term $\exp \left(A+\sum_{k=1}^{r} B_{k}\right)$ using the above upper bounds:

$$
\begin{aligned}
& \exp \left(A+\sum_{k=1}^{r} B_{k}\right) \leq e^{A} e^{r / 2} \prod_{i=1}^{r} \sqrt{\frac{2 \tau_{k}+3}{1-\left(d_{0}+k\right) /\left(2 \tau_{k}+3\right)}} \\
& \leq\left(1-\frac{d_{0}}{2 \tau_{0}+3}\right)^{-\left(\tau_{0}+3 / 2\right)+d_{0} / 2} \exp \left(-\frac{d_{0}}{2}+\frac{\left(d_{0}+r\right)^{2}}{8 t+4}+\frac{\left(d_{0}+r\right)^{3}}{4(2 t+1)^{2}}\right) \\
& \quad \times\left(\frac{2 t_{0}+3}{2 t+1}\right)^{d_{0} / 2}(2 t+1)^{-r / 2} \prod_{i=1}^{r} \sqrt{\frac{2 \tau_{k}+3}{1-\left(d_{0}+k / 2 \tau_{k}+3\right)}} .
\end{aligned}
$$

Using the above upper bound, we obtain

$$
p_{\tau} \leq C\left(r, d_{0}, t_{0}, t\right) \prod_{k=1}^{r}\left[\left(2 \tau_{k}+3-\left(d_{0}+k\right)\right)^{-1 / 2}\left(1+\frac{1}{\tau_{k}+1 / 2}\right)\right],
$$

where

$$
\begin{aligned}
& C\left(r, d_{0}, t_{0}, t\right)=\frac{\left(d_{0}+r-1\right) !}{\left(d_{0}-1\right) !}\left(1-\frac{d_{0}}{2 \tau_{0}+3}\right)^{-\left(\tau_{0}+3 / 2\right)+d_{0} / 2} \\
& \quad \times \exp \left(-\frac{d_{0}}{2}+\frac{\left(d_{0}+r\right)^{2}}{8 t+4}+\frac{\left(d_{0}+r\right)^{3}}{4(2 t+1)^{2}}\right)\left(\frac{2 t_{0}+3}{2 t+1}\right)^{d_{0} / 2}(2 t+1)^{-r / 2} .
\end{aligned}
$$


We need to sum over all possible insertion times to bound the probability $p^{*}$ of interest. We set $\tau_{k}^{\prime} \leftarrow \tau_{k}-\left\lceil\left(d_{0}+k\right) / 2\right\rceil$ for $k=1, \ldots, r$. For $d=o(\sqrt{t})$ and $r=o\left(t^{2 / 3}\right)$, we obtain

$$
\begin{aligned}
& p^{*} \leq C\left(r, d_{0}, t_{0}, t\right) \sum_{\substack{t_{0}+1 \leq \tau_{1} \\
<\cdots<\tau_{r} \leq t}} \prod_{k=1}^{r}\left[\left(2 \tau_{k}+3-\left(d_{0}+k\right)\right)^{-1 / 2}\left(1+\frac{1}{\tau_{k}+1 / 2}\right)\right] \\
& \leq C\left(r, d_{0}, t_{0}, t\right) \\
& \times \sum_{\substack{t_{0}-\left\lceil d_{0} / 2\right\rceil+1 \leq \tau_{1}^{\prime} \\
\leq \cdots \leq \tau_{r}^{\prime} \leq t-\left\lceil\left(d_{0}+r\right) / 2\right\rceil}} \prod_{k=1}^{r}\left[\left(2 \tau_{k}^{\prime}+3\right)^{-1 / 2}\left(1+\frac{1}{\tau_{k}^{\prime}+\left(d_{0}+k\right) / 2+1 / 2}\right)\right] \\
& \leq \frac{C\left(r, d_{0}, t_{0}, t\right)}{r !}\left(\sum_{t_{0}-\left\lceil d_{0} / 2\right\rceil}^{t-\left\lceil\left(d_{0}+r\right) / 2\right\rceil}\left(2 \tau_{k}^{\prime}+3\right)^{-1 / 2}+\frac{1}{\sqrt{2}}\left(\tau_{k}^{\prime}+\frac{3}{2}\right)^{-3 / 2}\right)^{r} \\
& \leq \frac{C\left(r, d_{0}, t_{0}, t\right)}{r !}\left(\int_{0}^{t-(d+r) / 2}\left[(2 x+3)^{-1 / 2}+\frac{1}{\sqrt{2}}\left(x+\frac{3}{2}\right)^{-3 / 2}\right] \mathrm{d} x\right)^{r} \\
& \leq \frac{C\left(r, d_{0}, t_{0}, t\right)}{r !}\left(\sqrt{2 t+3-\left(d_{0}+r\right)}+\frac{2}{3}\right)^{r} \\
& \leq \frac{C\left(r, d_{0}, t_{0}, t\right)}{r !}(2 t)^{r / 2} \exp \left(-\frac{r}{2} \frac{d_{0}+r-3}{2 t}\right) \exp \left(\frac{2 r}{3 \sqrt{2 t-\left(d_{0}+r\right)+3}}\right) \\
& \leq\left(\begin{array}{c}
d_{0}+r-1 \\
d_{0}-1
\end{array}\right)\left(\frac{2 t_{0}+3}{2 t+1}\right)^{d_{0} / 2}\left[\left(1-\frac{d_{0}}{2 t_{0}+3}\right)^{-\left(1-d_{0} /\left(2 t_{0}+3\right)\right)}\right]^{t_{0}+3 / 2} \\
& \times\left(\frac{2 t}{2 t+1}\right)^{r / 2} \exp \left(-\frac{d_{0}}{2}+\frac{\left(d_{0}+r\right)^{2}}{8 t+4}+\frac{\left(d_{0}+r\right)^{3}}{4(2 t+1)^{2}}-\frac{r\left(d_{0}+r-3\right)}{4 t}\right. \\
& \left.+\frac{2 r}{3 \sqrt{2 t+3-\left(d_{0}+r\right)}}\right) \text {. }
\end{aligned}
$$

By removing the $o(1)$ terms in the exponential and using the fact that $x^{-x} \leq e$, we obtain the following bound on the probability $p^{*}$ :

$$
p^{*} \leq\left(\begin{array}{c}
d_{0}+r-1 \\
d_{0}-1
\end{array}\right)\left(\frac{2 t_{0}+3}{2 t+1}\right)^{d_{0} / 2} \exp \left(\frac{3}{2}+t_{0}-\frac{d_{0}}{2}+\frac{2 r}{3 \sqrt{t}}\right),
$$

completing the proof.

We now restate and prove Lemma 4.7 .

Lemma 4.7. The $k$ highest degrees satisfy $\Delta_{i} \leq \Delta_{i-1}-\sqrt{t} / f(t)$ whp, $i=1, \ldots, k$. 
Proof. Let $\mathcal{A}_{4}$ denote the event that there are two vertices among the first $t_{1}$ with degree at least $t_{0}^{-1} t^{1 / 2}$ and within $\sqrt{t} / f(t)$ of each other. By the definition of conditional probability and Lemma 4.4, we have

$$
\operatorname{Pr}\left[\mathcal{A}_{4}\right]=\operatorname{Pr}\left[\mathcal{A}_{4} \mid \overline{\mathcal{A}_{3}}\right] \operatorname{Pr}\left[\overline{\mathcal{A}_{3}}\right]+\operatorname{Pr}\left[\mathcal{A}_{4} \mid \mathcal{A}_{3}\right] \operatorname{Pr}\left[\mathcal{A}_{3}\right] \leq \operatorname{Pr}\left[\mathcal{A}_{4} \mid \overline{\mathcal{A}_{3}}\right]+o(1) .
$$

It suffices to show that $\operatorname{Pr}\left[\mathcal{A}_{4} \mid \overline{\mathcal{A}}_{3}\right]=o(1)$. Note that by a simple union bound, we have

$$
\operatorname{Pr}\left[\mathcal{A}_{4}\right] \leq \sum_{1 \leq s_{1}<s_{2} \leq t_{1}} \sum_{l=-\sqrt{t} / f(t)}^{\sqrt{t} / f(t)} p_{l, s_{1}, s_{2}}=O\left(t_{1}^{2} \frac{\sqrt{t}}{f(t)} \max p_{l, s_{1}, s_{2}}\right)
$$

where $p_{l, s_{1}, s_{2}}=\operatorname{Pr}\left[d_{t}\left(s_{1}\right)-d_{t}\left(s_{2}\right)=l \mid \overline{\mathcal{A}_{3}}\right]$.

We consider two cases, and we show that in both of them, $\max p_{l, s_{1}, s_{2}}=$ $o\left(f(t) / t_{1}^{2} \sqrt{t}\right)$.

Case 1: $\left(s_{1}, s_{2}\right) \notin E\left(G_{t}\right)$. Note that at time $t_{1}$, there exist $m_{t_{1}}=3 t_{1}+3<4 t_{1}$ edges in $G_{t_{1}}$ :

$$
\begin{aligned}
p_{l, s_{1}, s_{2}} & \sum_{r=t_{0}^{-1} t^{1 / 2}}^{t_{0}^{1 / 6} t^{1 / 2}} \sum_{d_{1}, d_{2}=3}^{4 t_{1}} \operatorname{Pr}\left[d_{t}\left(s_{1}\right)=r \wedge d_{t}\left(s_{2}\right)=r-l \mid d_{t_{1}}\left(s_{1}\right)=d_{1}, d_{t_{1}}\left(s_{2}\right)=d_{2}\right] \\
\leq & t_{0}^{1 / 6} t^{1 / 2} \sum_{d_{1}, d_{2}=3}^{4 t_{1}}\left(\begin{array}{c}
2 t_{0}^{1 / 6} t^{1 / 2} \\
d_{1}-1
\end{array}\right)\left(\begin{array}{c}
2 t_{0}^{1 / 6} t^{1 / 2} \\
d_{2}-1
\end{array}\right)\left(\frac{2 t_{0}+3}{2 t+1}\right)^{\left(d_{1}+d_{2}\right) / 2} \\
& \times \exp \left(\frac{3}{2}+t_{1}+\frac{2 t_{0}^{1 / 6}}{3}\right) \\
\leq & t_{0}^{1 / 6} t^{1 / 2} \sum_{d_{1}, d_{2}=3}^{4 t_{1}}\left(2 t_{0}^{1 / 6} t^{1 / 2}\right)^{d_{1}+d_{2}-2}\left(\frac{2 t_{0}+3}{2 t+1}\right)^{\left(d_{1}+d_{2}\right) / 2} \exp \left(2 t_{1}\right) \\
\leq & t_{0}^{1 / 6} t^{1 / 2} e^{2 t_{1}} t_{1}^{2}\left(2 t_{0}^{1 / 6} t^{1 / 2}\right)^{8 t_{1}-2}\left(\frac{2 t_{0}+3}{2 t+1}\right)^{4 t_{1}} \\
= & t_{0}^{4 t_{1} / 3+1 / 6} t^{-1 / 2} e^{2 t_{1}} t_{1}^{2} 2^{8 t_{1}}\left(2 t_{0}+3\right)^{4 t_{1}}\left(\frac{t}{2 t+1}\right)^{4 t_{1}}=o\left(\frac{f(t)}{t_{1}^{2} \sqrt{t}}\right)
\end{aligned}
$$

Note that we omitted the tedious calculation justifying the transition from (2) to (3), since calculating the upper bound of the joint probability distribution is very similar to the calculation of $\operatorname{Pr}\left[\mathcal{A}_{1}\right]$ in Lemma 4.2. 
Case 2: $\left(s_{1}, s_{2}\right) \in E\left(G_{t}\right)$. Observe that in any case, $\left(s_{1}, s_{2}\right)$ share at most two faces (which may change over time). Note that the two connected vertices $s_{1}, s_{2}$ share a common face only if $s_{1}, s_{2} \in\{1,2,3\} .{ }^{4}$ Consider the following modified process $\mathcal{Y}^{\prime}$ : whenever an incoming vertex "chooses" one of the two common faces, we do not insert it. We choose two other faces that are not common to $s_{1}, s_{2}$ and add one vertex in each of those. Notice that the number of faces increases by 1 for both $s_{1}, s_{2}$ as in the original process, and the difference of the degrees remains the same. An algebraic manipulation similar to that in Case 1 gives the desired result.

Acknowledgments. We would like to thank the reviewers for their detailed comments. Also, we would like to thank Luc Devroye for pointing out reference [Broutin and Devroye 06], and Alexis Darrasse for pointing out references [Albenque and Marckert 08, Panholzer and Seitz 10].

Funding. The authors' research was supported by NSF Grant No. CCF-1013110.

\section{References}

[Aiello et al. 01] W. Aiello, F. Chung, and L. Lu. "A Random Graph Model for Power Law Graphs." Experimental Mathematics 10 (2001), 53-66.

[Albenque and Marckert 08] M. Albenque and J. F. Marckert. "Some Families of Increasing Planar Maps." Electronic Journal of Probability 13 (2008), 1624-1671.

[Alon and Spencer 08] N. Alon and J. Spencer. The Probabilistic Method. WileyInterscience, 2008.

[Andrade and Miranda 05] R. F. S. Andrade and J. G. V. Miranda. "Spectral Properties of the Apollonian Network." Physica A 356, (2005), 1-5.

[Andrade et al. 05] J. S. Andrade, H. J. Herrmann, R. F. S. Andrade, and L. R. da Silva. "Apollonian Networks: Simultaneously Scale-Free, Small World Euclidean, Space Filling, and with Matching Graphs." Phys. Rev. Lett. 94 (2005), 018702.

[Azuma 67] K. Azuma. "Weighted Sums of Certain Dependent Variables." Tohoku Math. J. 3 (1967), 357-367.

[Barabási and Albert 99] A. Barabási and R. Albert. "Emergence of Scaling in Random Networks." Science 286 (1999), 509-512.

[Bodini et al. 07] O. Bodini, A. Darrasse, and M. Soria. "Distances in Random Apollonian Network Structures." arXiv abs/0712.2129, 2007.

[Bollobás 98] B. Bollobás. Modern Graph Theory. Springer, 1998.

[Bollobás and Riordan 04] B. Bollobás and O. Riordan. "The Diameter of a Scale-Free Random Graph." Combinatorica 24 (2004), 5-34.

\footnotetext{
${ }^{4}$ We analyze the case $s_{1}, s_{2} \geq 4$. The other case is treated in the same manner.
} 
[Bollobás et al. 01] B. Bollobás, O. Riordan, J. Spencer, and G. Tusnády. "The Degree Sequence of a Scale Free Random Graph Process." Random Struct. Algorithms 18 (2001), 279-290.

[Boyd 82] D. W. Boyd. "The Sequence of Radii of the Apollonian Packing." Mathematics of Computation 19 (1982), 249-254.

[Broutin and Devroye 06] N. Broutin and L. Devroye. "Large Deviations for the Weighted Height of an Extended Class of Trees." Algorithmica 46 (2006), 271-297.

[Chung et al. 03] F. Chung, L. Lu, and V. H. Vu. "Spectra of Random Graphs with Given Expected Degrees." Proc. Natl. Acad. Sci. USA 100 (2003), 6313-6318.

[Chung Graham 97] F. Chung Graham. Spectral Graph Theory. American Mathematical Society, 1997.

[Chung Graham and Lu 06] F. Chung Graham and L. Lu. Complex Graphs and Networks. American Mathematical Society, 2006.

[Cooper and Frieze 03] C. Cooper A. and A. Frieze. "A General Model of Web Graphs." Random Structures and Algorithms 22 (2003), 311-335.

[Cooper and Uehara 10] C. Cooper and R. Uehara. "Scale Free Properties of Random k-Trees." Mathematics in Computer Science 3 (2010), 489-496.

[Darrasse and Soria 07] A. Darrasse and M. Soria. "Degree Distribution of Random Apollonian Network Structures and Boltzmann Sampling." In 2007 Conference on Analysis of Algorithms, AofA 0\%, DMTCS Proceedings, pp. 313-324, 2007.

[Darrasse et al. 10] A. Darrasse, H.-K. Hwang, O. Bodini, and M. Soria. "The Connectivity-Profile of Random Increasing $k$-Trees." In $A N A L C O, 99-106$. SIAM, 2010.

[Doye and Massen 05] J. P. K Doye and C. P. Massen. "Self-Similar Disk Packings as Model Spatial Scale-Free Networks." Phys. Rev. E 71 (2005) 016128.

[Ebrahimzadeh et al. 13] E. Ebrahimzadeh, L. Farczadi, P. Gao, A. Mehrabian, C. M. Sato, N. Wormald, and J. Zung. "On the Longest Paths and the Diameter in Random Apollonian Networks." Electron. Notes Discrete Math. 43 (2013), 355-365.

[Fabrikant et al. 02] A. Fabrikant, E. Koutsoupias, and C. Papadimitriou. "Heuristically Optimized Trade-offs." In Proceedings of the 29th International Colloquium on Automata, Languages, and Programming (ICALP), pp. 110-122, 2002.

[Flaxman et al. 05] A. Flaxman, A. Frieze, and T. Fenner. "High Degree Vertices and Eigenvalues in the Preferential Attachment Graph." Internet Mathematics 2 (2005), $1-19$.

[Gao 09] Y. Gao. "The Degree Distribution of Random $k$-Trees." Theoretical Computer Science 410 (2009), 688-695.

[Gao and Hobson 06] Y. Gao and C. Hobson. "Random k-Tree as a Model for Complex Networks." In Workshop on Algorithms and Models for the Web-Graph (WAW), 2006.

[Graham et al. 03] R. L. Graham, J. C. Lagarias, C. L. Mallows, A. R. Wilks, and C. H. Yan. "Apollonian Circle Packings: Number Theory." J. Number Theory 100 (2003), $1-45$. 
[Hoeffding 63] W. Hoeffding. "Probability Inequalities for Sums of Bounded Random Variables." J. Amer. Statist. Assoc. 58 (1963), 13-30.

[Klein 14] P. Klein. "Optimization Algorithms for Planar Graphs." Available online http://planarity.org/, 2014.

[Kloks 94] T. Kloks. Treewidth: Computations and Approximations. Springer, 1994.

[Leskovec and Faloutsos 07] J. Leskovec and C. Faloutsos. "Scalable Modeling of Real Graphs Using Kronecker Multiplication." In Machine Learning Proceedings of the Twenty-Fourth International Conference (ICML 2007), Corvalis, Oregon, USA, June 20-24, 2007, pp. 497-504, 2007.

[Mihail and Papadimitriou 02] M. Mihail and C. Papadimitriou. "On the Eigenvalue Power Law." RANDOM '02, pp. 254-262, 2002.

[Panholzer and Seitz 10] A. Panholzer and G. Seitz. "Ordered Increasing $k$-Trees: Introduction and Analysis of a Preferential Attachment Network Model." DMTCS Proc. AofA '10, pp. 549-564, 2010.

[Pralat and Wormald 07] P. Pralat and N. Wormald. "Growing Protean Graphs." Internet Mathematics 4 (2007), 1-16.

[Strang 05] G. Strang. Linear Algebra and Its Applications Brooks Cole, 2005.

[Wu et al. 06] Z.-X. Wu, X.-J. Xu, and Y.-H. Wang. "Comment on 'Maximal Planar Networks with Large Clustering Coefficient and Power-Law Degree Distribution."'” Physical Review E 73 (2006), 058101.

[Zhang et al. 06] Z. Z. Zhang, F. Comellas, G. Fertin, and L. Rong. "High Dimensional Apollonian Networks." J. Phys. A-Math. Gen. 39 (2006), 1811-1818.

[Zhou et al. 05] T. Zhou, G. Yan, and B. H. Wang. "Maximal Planar Networks with Large Clustering Coefficient and Power-Law Degree Distribution." Phys. Rev. E 71 (2005), 046141.

Alan Frieze, Department of Mathematical Sciences, Carnegie Mellon University, 5000 Forbes Avenue, Pittsburgh, PA 15213, USA (af1p@random.math.cmu.edu)

Charalampos E. Tsourakakis, Computer and Information Science Department, University of Pennsylvania, 5538 Hobart Street, Pittsburgh, PA 15217, USA (ctsourak@math. cmu.edu) 